\title{
English history teachers' views on what substantive content young people should be taught
}

Article

Accepted Version

Harris, R. and Burn, K. (2016) English history teachers' views on what substantive content young people should be taught. Journal of Curriculum Studies, 48 (4). pp. 518-546. ISSN 1366-5839 doi:

https://doi.org/10.1080/00220272.2015.1122091 Available at https://centaur.reading.ac.uk/49559/

It is advisable to refer to the publisher's version if you intend to cite from the work. See Guidance on citing.

To link to this article DOI: http://dx.doi.org/10.1080/00220272.2015.1122091

Publisher: Taylor \& Francis

All outputs in CentAUR are protected by Intellectual Property Rights law, including copyright law. Copyright and IPR is retained by the creators or other copyright holders. Terms and conditions for use of this material are defined in the End User Agreement.

www.reading.ac.uk/centaur 
Central Archive at the University of Reading

Reading's research outputs online 
Table 1 - There is an appropriate proportion of British history within the proposed curriculum

\begin{tabular}{|c|c|c|c|c|c|c|c|c|c|c|c|}
\hline & $\begin{array}{l}\text { Strongly } \\
\text { agree }\end{array}$ & $\%$ & Agree & $\%$ & Neither & $\%$ & Disagree & $\%$ & $\begin{array}{l}\text { Strongly } \\
\text { disagree }\end{array}$ & & Total \\
\hline Comprehensive & 3 & $1.2 \%$ & 19 & $7.6 \%$ & 22 & $8.8 \%$ & 85 & $34.1 \%$ & 120 & $48.2 \%$ & 249 \\
\hline Grammar & 0 & $0.0 \%$ & 1 & $9.1 \%$ & 0 & $0.0 \%$ & 7 & $63.6 \%$ & 3 & $27.3 \%$ & 11 \\
\hline Independent & 2 & $4.4 \%$ & 8 & $17.8 \%$ & 2 & $4.4 \%$ & 14 & $31.1 \%$ & 19 & $42.2 \%$ & 45 \\
\hline Academy & 1 & $3.8 \%$ & 3 & $11.5 \%$ & 2 & $7.7 \%$ & 10 & $38.5 \%$ & 10 & $38.5 \%$ & 26 \\
\hline New Academy & 1 & $0.5 \%$ & 26 & $12.7 \%$ & 28 & $13.7 \%$ & 59 & $28.9 \%$ & 90 & $44.1 \%$ & 204 \\
\hline Sixth form & 0 & $0.0 \%$ & 1 & $10.0 \%$ & 1 & $10.0 \%$ & 3 & $30.0 \%$ & 5 & $50.0 \%$ & 10 \\
\hline Special needs & 0 & $0.0 \%$ & 1 & $100.0 \%$ & 0 & $0.0 \%$ & 0 & $0.0 \%$ & 0 & $0.0 \%$ & 1 \\
\hline $\begin{array}{l}\text { All teachers of } \\
\text { ages } 11-14\end{array}$ & 7 & $1.3 \%$ & 58 & $10.8 \%$ & 54 & $10.1 \%$ & 175 & $32.6 \%$ & 242 & $45.1 \%$ & 536 \\
\hline
\end{tabular}

Table 2 - There is an appropriate proportion of European and world history in the proposed curriculum

\begin{tabular}{|c|c|c|c|c|c|c|c|c|c|c|c|}
\hline & $\begin{array}{l}\text { Strongly } \\
\text { agree }\end{array}$ & $\%$ & Agree & $\%$ & Neither & $\%$ & Disagree & $\%$ & $\begin{array}{l}\text { Strongly } \\
\text { disagree }\end{array}$ & $\%$ & Total \\
\hline Comprehensive & 2 & $0.8 \%$ & 10 & $4.0 \%$ & 17 & $6.8 \%$ & 88 & $35.2 \%$ & 133 & $53.2 \%$ & 250 \\
\hline Grammar & 0 & $0.0 \%$ & 0 & $0.0 \%$ & 1 & $9.1 \%$ & 3 & $27.3 \%$ & 7 & $63.6 \%$ & 11 \\
\hline Independent & 0 & $0.0 \%$ & 6 & $13.0 \%$ & 1 & $2.2 \%$ & 19 & $41.3 \%$ & 20 & $43.5 \%$ & 46 \\
\hline Academy & 1 & $3.8 \%$ & 3 & $11.5 \%$ & 0 & $0.0 \%$ & 14 & $53.8 \%$ & 8 & $30.8 \%$ & 26 \\
\hline New Academy & 0 & $0.0 \%$ & 14 & $6.8 \%$ & 26 & $12.6 \%$ & 66 & $31.9 \%$ & 101 & $48.8 \%$ & 207 \\
\hline Sixth form & 0 & $0.0 \%$ & 1 & $10.0 \%$ & 0 & $0.0 \%$ & 2 & $20.0 \%$ & 7 & $70.0 \%$ & 10 \\
\hline Special needs & 0 & $0.0 \%$ & 1 & $100.0 \%$ & 0 & $0.0 \%$ & 0 & $0.0 \%$ & 0 & $0.0 \%$ & 1 \\
\hline $\begin{array}{l}\text { All teachers of } \\
\text { ages } 11-14\end{array}$ & 3 & $0.6 \%$ & 34 & $6.3 \%$ & 45 & $8.3 \%$ & 190 & $35.1 \%$ & 269 & $49.7 \%$ & 541 \\
\hline
\end{tabular}


Table 3 - There is an appropriate balance between political, economic, social, religious and cultural history in the proposed curriculum.

\begin{tabular}{|c|c|c|c|c|c|c|c|c|c|c|c|}
\hline & $\begin{array}{l}\text { Strongly } \\
\text { agree }\end{array}$ & $\%$ & Agree & $\%$ & Neither & $\%$ & Disagree & $\%$ & $\begin{array}{l}\text { Strongly } \\
\text { disagree }\end{array}$ & $\%$ & Total \\
\hline Comprehensive & 1 & $0.4 \%$ & 20 & $8.1 \%$ & 20 & $8.1 \%$ & 92 & $37.1 \%$ & 115 & $46.4 \%$ & 248 \\
\hline Grammar & 0 & $0.0 \%$ & 0 & $0.0 \%$ & 2 & $18.2 \%$ & 5 & $45.5 \%$ & 4 & $36.4 \%$ & 11 \\
\hline Independent & 1 & $2.2 \%$ & 6 & $13.0 \%$ & 3 & $6.5 \%$ & 23 & $50.0 \%$ & 13 & $28.3 \%$ & 46 \\
\hline Academy & 0 & $0.0 \%$ & 3 & $12.0 \%$ & 4 & $16.0 \%$ & 8 & $32.0 \%$ & 10 & $40.0 \%$ & 25 \\
\hline New Academy & 0 & $0.0 \%$ & 22 & $10.7 \%$ & 24 & $11.7 \%$ & 67 & $32.7 \%$ & 92 & $44.9 \%$ & 205 \\
\hline Sixth form & 0 & $0.0 \%$ & 1 & $10.0 \%$ & 2 & $20.0 \%$ & 1 & $10.0 \%$ & 6 & $60.0 \%$ & 10 \\
\hline Special needs & 0 & $0.0 \%$ & 1 & $100.0 \%$ & 0 & $0.0 \%$ & 0 & $0.0 \%$ & 0 & $0.0 \%$ & 1 \\
\hline $\begin{array}{l}\text { All teachers of } \\
\text { ages } 11-14\end{array}$ & 2 & $0.4 \%$ & 52 & $9.7 \%$ & 53 & $9.9 \%$ & 195 & $36.4 \%$ & 234 & $43.7 \%$ & 536 \\
\hline
\end{tabular}

Table 4 - The amount of prescribed content in the proposed curriculum is just about right

\begin{tabular}{|c|c|c|c|c|c|c|c|c|c|c|c|}
\hline & $\begin{array}{l}\text { Strongly } \\
\text { agree }\end{array}$ & $\%$ & Agree & $\%$ & Neither & $\%$ & Disagree & $\%$ & $\begin{array}{l}\text { Strongly } \\
\text { disagree }\end{array}$ & Total & \\
\hline Comprehensive & 0 & $0.0 \%$ & 2 & $0.8 \%$ & 7 & $2.8 \%$ & 54 & $21.6 \%$ & 187 & $74.8 \%$ & 250 \\
\hline Grammar & 0 & $0.0 \%$ & 1 & $9.1 \%$ & 0 & $0.0 \%$ & 0 & $0.0 \%$ & 10 & $90.9 \%$ & 11 \\
\hline Independent & 0 & $0.0 \%$ & 3 & $6.5 \%$ & 0 & $0.0 \%$ & 15 & $32.6 \%$ & 28 & $60.9 \%$ & 46 \\
\hline Academy & 0 & $0.0 \%$ & 1 & $3.8 \%$ & 0 & $0.0 \%$ & 6 & $23.1 \%$ & 19 & $73.1 \%$ & 26 \\
\hline New Academy & 0 & $0.0 \%$ & 3 & $1.4 \%$ & 5 & $2.4 \%$ & 51 & $24.6 \%$ & 148 & $71.5 \%$ & 207 \\
\hline Sixth form & 0 & $0.0 \%$ & 0 & $0.0 \%$ & 2 & $20.0 \%$ & 2 & $20.0 \%$ & 6 & $60.0 \%$ & 10 \\
\hline Special needs & 0 & $0.0 \%$ & 1 & $100.0 \%$ & 0 & $0.0 \%$ & 0 & $0.0 \%$ & 0 & $0.0 \%$ & 1 \\
\hline $\begin{array}{l}\text { All teachers of } \\
\text { ages 11-14 }\end{array}$ & 0 & $0.0 \%$ & 11 & $2.0 \%$ & 12 & $2.2 \%$ & 126 & $23.3 \%$ & 392 & $72.5 \%$ & 541 \\
\hline
\end{tabular}

Table 5 - Covering history from the Stone Age to 1700 at Key Stage 2 will provide young people with a secure basis from which to go on learning history in Key Stage 3. 


\begin{tabular}{|c|c|c|c|c|c|c|c|c|c|c|c|}
\hline & $\begin{array}{l}\text { Strongly } \\
\text { agree }\end{array}$ & $\%$ & Agree & $\%$ & Neither & $\%$ & Disagree & $\%$ & $\begin{array}{l}\text { Strongly } \\
\text { disagree }\end{array}$ & Total & \\
\hline Comprehensive & 0 & $0.0 \%$ & 3 & $1.2 \%$ & 7 & $2.8 \%$ & 38 & $15.3 \%$ & 201 & $80.7 \%$ & 249 \\
\hline Grammar & 0 & $0.0 \%$ & 0 & $0.0 \%$ & 0 & $0.0 \%$ & 0 & $0.0 \%$ & 10 & $90.9 \%$ & 11 \\
\hline Independent & 1 & $2.1 \%$ & 3 & $6.4 \%$ & 3 & $6.4 \%$ & 13 & $27.7 \%$ & 27 & $57.4 \%$ & 47 \\
\hline Academy & 0 & $0.0 \%$ & 1 & $3.8 \%$ & 1 & $3.8 \%$ & 5 & $19.2 \%$ & 19 & $73.1 \%$ & 26 \\
\hline New Academy & 0 & $0.0 \%$ & 8 & $3.8 \%$ & 7 & $3.4 \%$ & 42 & $20.2 \%$ & 151 & $72.6 \%$ & 208 \\
\hline Sixth form & 0 & $0.0 \%$ & 0 & $0.0 \%$ & 1 & $10.0 \%$ & 2 & $20.0 \%$ & 7 & $70.0 \%$ & 10 \\
\hline Special needs & 0 & $0.0 \%$ & 0 & $0.0 \%$ & 0 & $0.0 \%$ & 1 & $100.0 \%$ & 0 & $0.0 \%$ & 1 \\
\hline $\begin{array}{l}\text { All teachers of } \\
\text { ages } 11-14\end{array}$ & 1 & $0.2 \%$ & 15 & $2.8 \%$ & 18 & $3.3 \%$ & 99 & $18.3 \%$ & 408 & $75.4 \%$ & 541 \\
\hline
\end{tabular}

Table 6 - Teaching the chronological sequence of events as set out in the content of the curriculum proposal will help young people to develop a clear historical framework or map of the past.

\begin{tabular}{|c|c|c|c|c|c|c|c|c|c|c|c|}
\hline & $\begin{array}{l}\text { Strongly } \\
\text { agree }\end{array}$ & $\%$ & Agree & $\%$ & Neither & $\%$ & Disagree & $\%$ & $\begin{array}{l}\text { Strongly } \\
\text { disagree }\end{array}$ & & Total \\
\hline Comprehensive & 1 & $0.4 \%$ & 39 & $15.5 \%$ & 22 & $8.8 \%$ & 72 & $28.7 \%$ & 117 & $46.6 \%$ & 251 \\
\hline Grammar & 1 & $9.1 \%$ & 2 & $18.2 \%$ & 0 & $0.0 \%$ & 3 & $27.3 \%$ & 5 & $45.5 \%$ & 11 \\
\hline Independent & 3 & $6.7 \%$ & 8 & $17.8 \%$ & 1 & $2.2 \%$ & 17 & $37.8 \%$ & 16 & $35.6 \%$ & 45 \\
\hline Academy & 0 & $0.0 \%$ & 3 & $12.0 \%$ & 4 & $16.0 \%$ & 10 & $40.0 \%$ & 8 & $32.0 \%$ & 25 \\
\hline New Academy & 8 & $3.9 \%$ & 41 & $19.8 \%$ & 17 & $8.2 \%$ & 66 & $31.9 \%$ & 75 & $36.2 \%$ & 207 \\
\hline Sixth form & 0 & $0.0 \%$ & 3 & $30.0 \%$ & 3 & $30.0 \%$ & 2 & $20.0 \%$ & 2 & $20.0 \%$ & 10 \\
\hline Special needs & 0 & $0.0 \%$ & 0 & $0.0 \%$ & 0 & $0.0 \%$ & 1 & $100.0 \%$ & 0 & $0.0 \%$ & 1 \\
\hline $\begin{array}{l}\text { All teachers of } \\
\text { ages } 11-14\end{array}$ & 13 & $2.4 \%$ & 93 & $17.2 \%$ & 44 & $8.1 \%$ & 169 & $31.3 \%$ & 221 & $40.9 \%$ & 540 \\
\hline
\end{tabular}

